\title{
Harmonic Analysis of Environmental Time Series with Missing Data or Irregular Sample Spacing
}

by Shabnam Dilmaghani ${ }^{a}$, Isaac C. Henry, Puripus Soonthornnonda ${ }^{b}$, Erik R. Christensen ${ }^{b}$, and Ronald C. Henry ${ }^{a *}$,

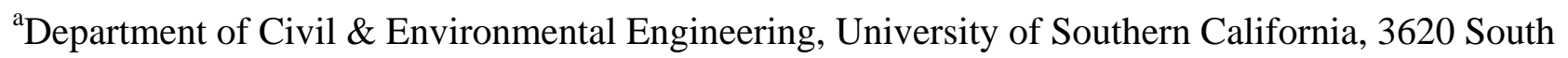
Vermont Avenue, Los Angeles, CA 90089-2541.

${ }^{\mathrm{b}}$ Department of Civil Engineering and Mechanics, University of Wisconsin-Milwaukee,

Milwaukee, WI 53201-0784

*Communicating author.

Two supplementary figures 


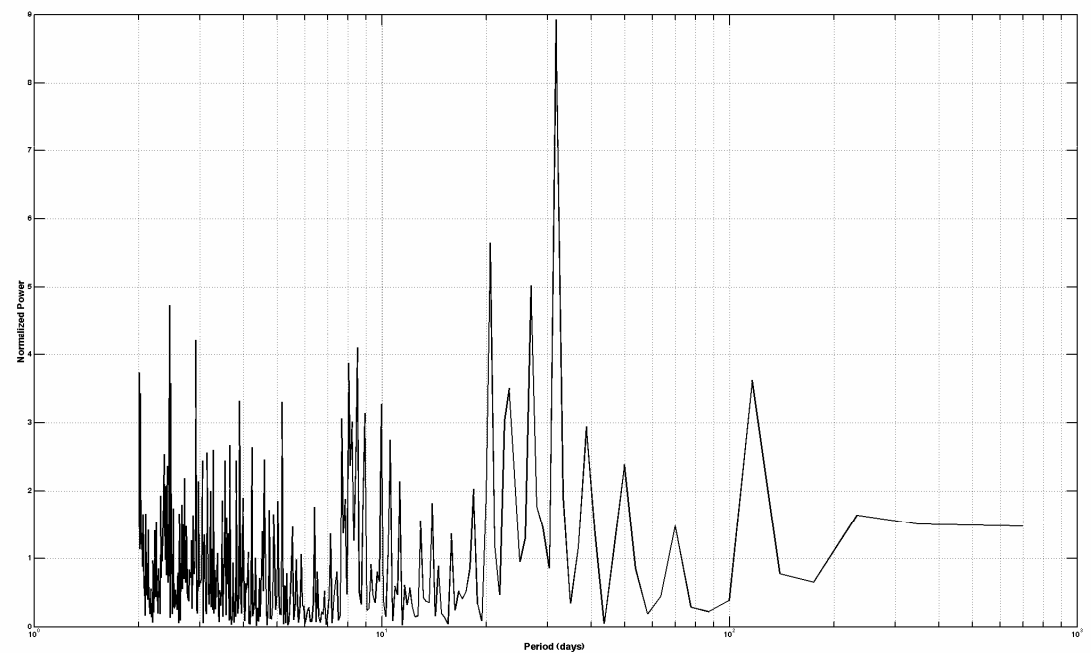

Figure S1. The Shuster periodogram of the zero filled daily copper concentrations calculated using the fast Fourier transform. Compare to the Lomb periodogram calculated for the same periods in Fig S2. The agreement for periods greater than 10 days is very good; understandably, the agreement for periods less than 10 days is not as good.

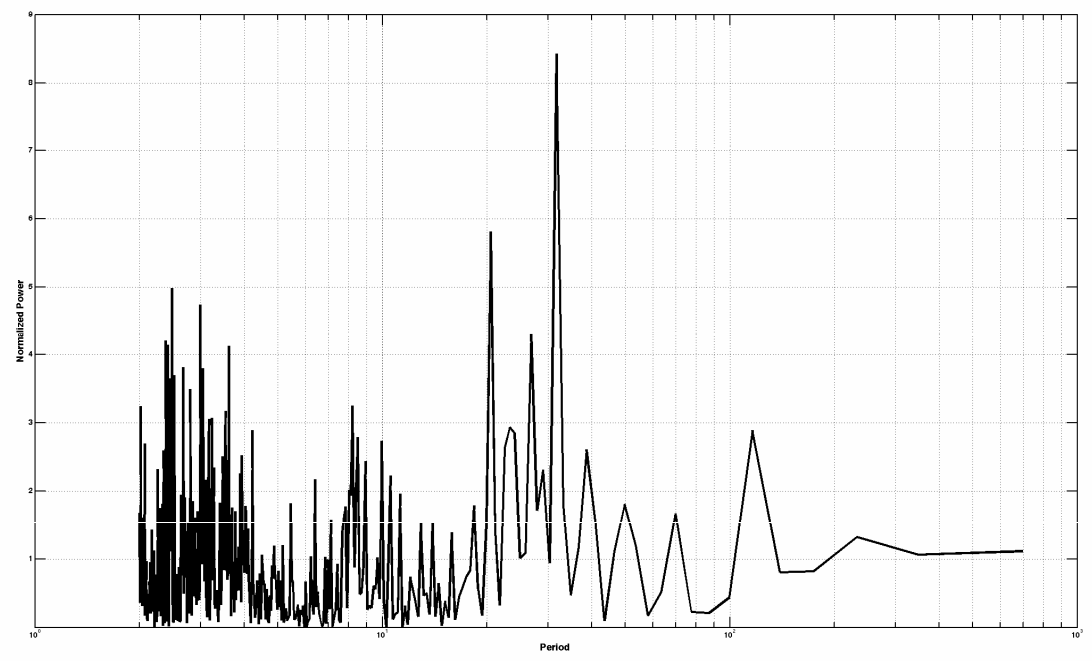

Figure S2. Lomb periodogram for the same frequencies as Fig. S1. 